\title{
WILEY-VCH
}

DOI: 10.1002/ ((please add manuscript number))

Article type: Full Paper

\section{Biohybrid Microtube Swimmers Driven by Single Captured Bacteria}

Morgan M. Stanton ${ }^{\dagger}$, Byung-Wook Park ${ }^{\dagger}$, Albert Miguel-López, Xing Ma, Metin Sitti*, Samuel Sánchez*

These authors contributed equally to this work.

Dr. M.M. Stanton, Dr. B-W. Park, and Prof. M. Sitti,

Max Planck Institute for Intelligent Systems, Heisenbergstraße 3, 70569 Stuttgart, Germany

E-mail: sitti@is.mpg.de

Dr. X. Ma

School of Materials Science and Engineering, Harbin Institute of Technology Shenzhen Graduate School, 518055 Shenzhen, China

A. Miguel-López and Prof. S. Sánchez,

Institució Catalana de Recerca i EstudisAvancats (ICREA), Pg. Lluís Companys 23, 08010

Barcelona, Spain

Institut de Bioenginyeria de Catalunya (IBEC), Baldiri i Reixac 10-12, 08028 Barcelona, Spain

E-mail: ssanchez@ibecbarcelona.eu

Keywords: biohybrid, polydopamine, micromotor, microswimmer, E. coli

Bacteria biohybrids employ the motility and power of swimming bacteria to carry and maneuver microscale particles. They have the potential to perform micro- drug and cargo deliver in vivo, but have been limited by poor design, reducing swimming capabilities and impeding functionality. To address this challenge, motile Escherichia coli (E. coli), were captured inside electropolymerized microtubes, exhibiting the first report of a bacteria microswimmer that does not utilize a spherical particle chassis. Single bacterium became partially trapped within the tube and became a bioengine to push the microtube though biological media. Microtubes were modified with 'smart' material properties for motion control, including a bacteria-attractant polydopamine inner layer, addition of magnetic components for external guidance, and a biochemical kill trigger to cease bacterium swimming on demand. Swimming dynamics of the bacteria biohybrid were quantified by comparing 


\section{WILEY-VCH}

'length of protrusion' (LOP) of bacteria from the microtubes with respect to changes in angular autocorrelation and swimmer mean square displacement (MSD). The multifunctional microtubular swimmers present a new generation of biocompatible micromotors towards future micro-biorobots and minimally invasive medical applications.

\section{Introduction}

Biohybrid microsystems are the integration of bioactuators with artificial materials; ${ }^{[1-3]}$ they exploit the motility and sensing capability of biological cells for generating functional micro-machines. Unlike other types of micromotors, biohybrid motors are powered by natural, non-toxic fuel sources found in biological media ${ }^{[4]}$ and operate by converting chemical energy into work. ${ }^{[5]}$ Due to the biocompatibility of their power source, biohybrid microsystems have the potential to operate in in vivo environments for biomedical drug and cargo delivery and micromanipulation of cell tissue. The miniaturized biohybrid motors aim to achieve what macro-scale medical tools currently perform, but at the micron scale, making them less invasive and capable of functioning in smaller spaces. Biohybrids combine material properties, bioactuation, and biosensing in a single motor making them more apt for advanced biomedical applications than chemically powered microswimmers that do not possess these advantageous features. ${ }^{[6]}$ Significant advancements have been made in designing biohybrid microswimmers capable of multiple tasks, ${ }^{[7-11]}$ but the next generation of micromotors must overcome significant challenges, including directional guidance, impaired swimming due to cargo load, and trigger-activated response. Moreover, these challenges should be solved by multifunctional biohybrid micromotor systems for facile performance of multiple programmed tasks. The power and sensing capabilities of motile bacteria are optimal for creating a swimming biohybrid to meet these demands on the few microns length scale, where 'smart' material properties can assist in localized cell integration and improve swimming efficiency.

Bacteria occupy multiple areas of the human microbiome ${ }^{[12]}$ and are an ideal candidate for powering and guiding biohybrid microswimmers. ${ }^{[13]}$ Bacteria flagellar motion has been 


\section{WILEY-VCH}

utilized powering microrotors $^{[14,15]}$ and for the delivery of nanoliposomes, ${ }^{[16-18]}$ but the largest portion of bacteria-based biohybrid systems research has investigated bacteria-driven particle microswimmers, ${ }^{[19-25]}$ where single or multiple bacteria adhere to a spherical particle and carry it while swimming. For these systems, selective patterning of the particle with metals ${ }^{[26]}$ or plasma etching ${ }^{[27]}$ facilitates bacteria adhesion and consequently improves directional swimming. However, bacteria attachment to a particle induces torque and rotational motion, decreasing the net translational motion. ${ }^{[28]}$ Other types of motor bodies for bacteria attachment that minimize rotational motion need to be investigated to improve bacteria microswimmer performance. This study is the first attempt at integrating a single motile bacterium with a microtube and a synthetic material that is not a spherical particle. Microtubular chassis have proven advantageous for sperm-driven biohybrid swimmers (spermbots) and provide highly directional swimming and accurate cargo delivery, ${ }^{[29-31]}$ but spermnbots are limited to only infertility treatment applications and are large scale microswimmers (60 - $70 \mu \mathrm{m}$ long). Bacteria-driven swimmers can exploit the advantages of microtubular systems, while functioning at significantly smaller length scales $(\sim 10 \mu \mathrm{m})$ than the smallest reported spermbot $^{[32]}$ and could have a much wider range of biomedical applications in different fluid regions of the human body, including the small and large intestine. Bacteria motility, rapid doubling rate, and ubiquitous presence in the human body mean bacteria should be further investigated for their ability to power micro-biorobots.

For the reported biohybrid, Escherichia coli (E. coli), were chosen to act as the microswimmers' bioengines. E. coli are one of the most well studied bacteria systems and are naturally found in the human lower gastrointestinal tract ${ }^{[33]}$. They have multiple rotating flagella that can bundle to propel them in solution or unbundle for directional reorientation for a swimming mechanism known as 'run and tumble'. ${ }^{[34]}$ Capture of an E. coli bacterium within a microtube would be a model proof-of-concept biohybrid for understanding the first bacteriamicrotube swimmer. To accomplish this goal, a single motile E. coli bacterium was partially 


\section{WILEY-VCH}

bound to the inside of a polydopamine (PDA) modified microtube and was powered by the motile bacterium flagella. The microtube fabrication process tailored the tube length to match the bacteria size to improve the swimming efficiency, incorporated biofunctionalization for bacteria capture, and included a triggering mechanism to inhibit swimming. Once the E. coli was coupled to the microtube, depth of penetration of the bacterium into the tube was used to analyze and understand the swimming behavior of the biohybrid. The microswimmer demonstrates multi-functionality that has not been observed in other bacteria-driven swimmers, including guided cell adhesion, magnetic steerability, and a chemically activated termination switch. The presented biohybrid microswimmer demonstrates the significant advantages of embracing microtubular swimmers and presents a new generation of biocompatible micromotors towards future biomedical applications.

\section{Results and Discussion}

\subsection{Biohybrid Fabrication and Characterization}

The main body of the conical microtube was fabricated with electropolymerized polypyrrole (Ppy) deposited on a Au-coated polycarbonate membrane. The electrodeposition process permitted precise control of the polymer thickness for the tube. After Ppy deposition, microtubes were modified internally with an adsorbed nanosized layer of PDA (two layers). An additional layer of $\mathrm{Ni}$ nanoparticles (three layers) for magnetic guidance or urease to act as a chemical trigger could also be added to increase the functionality of the system. For both sets of tubes, PDA occupied the most inner layer. A schematic for the microtube fabrication can be seen in Figure 1A. The positively-charged, internal PDA coating acted as an attractant to lure the negatively-charged E. coli and without the PDA layer, E. coli did not attach to the tube. PDA is a robust polymer naturally occurring in the adhesive proteins in mussels ${ }^{[35]}$ and has the

ability to immobilize and adhere bacteria while maintaining their viability ${ }^{[36,37]}$. Unlike other bacteria adhesive polymers, such as poly-L-lysine, ${ }^{[38]}$ PDA adheres strongly to surfaces and 


\section{WILEY-VCH}

retains its structure even after exposure to solvents, making it ideal for robust and stable bacteria integration. The bacteria displayed limited or no adhesive attraction to the outer tube layer of Ppy. For motility, a single bacterium became partially trapped on the PDA within the tube and became a bioengine to push the microtube through solution, generating a biohybrid swimmer with an average velocity of $5 \pm 1 \mu \mathrm{m} / \mathrm{s}$ as seen in Figure 1B (also in Videos SV1, SV2, Supporting Information). The conical Ppy tubes are approximately $8-11 \mu \mathrm{m}$ long with the diameter of the narrow end between 1.0 and $1.5 \mu \mathrm{m}$ and diameter of the wider end at $2.0 \mu \mathrm{m}$ (Figure 2A). The E. coli bacteria have a body length of $3-5 \mu \mathrm{m}$ and a diameter between 500 and $600 \mathrm{~nm}$, making them an optimal fit for the microtube. Polymerization of dopamine on the Ppy tube yielded a nanometer thick layer of PDA with defined roughness that was confined to the inner walls of the microtube (Figure 2B). If a three-layer tube was fabricated, Ni nanoparticles were electrodeposited (Figure 2C) first on the Ppy, followed by adsorption of PDA on the Pyr-Ni surface.

The Ppy, Pyr-Ni, and subsequent PDA deposition was confirmed with X-ray photoelectron spectroscopy (XPS) (Figure 2D) with the spectra of the C 1s, O 1s, and N 1s shown in Figure 2E. For Ppy, the $\mathrm{C}$ 1s peak can be decomposed into three different components located at $284 \mathrm{eV}(\mathrm{C}-\mathrm{C}, \mathrm{C}=\mathrm{C}), 286 \mathrm{eV}(\mathrm{C}-\mathrm{N})$, and $288 \mathrm{eV}(\mathrm{C}=\mathrm{N})$. The $\mathrm{N} 1 \mathrm{~s}$ core level spectra of Ppy is dominated by a main peak at $400 \mathrm{eV}$, assigned to uncharged amine nitrogen of the polymer, the shoulder contribution at $398 \mathrm{eV}$ is attributed to deprotonated uncharged imine nitrogen atoms, and the $402 \mathrm{eV}$ peak indicates protonated nitrogen atoms. After deposition of nickel layer, peaks located at $856 \mathrm{eV}$ and $873 \mathrm{eV}$ with corresponding satellite peaks at $861 \mathrm{eV}$ and $879 \mathrm{eV}$ were observed and attributed to Ni $2 \mathrm{p}_{3 / 2}$ and Ni $2 \mathrm{p}_{1 / 2}$ spinorbit levels of $\mathrm{NiO}$, respectively (Figure 2D). After PDA deposition, the $\mathrm{NiO}$ peaks were no longer observable with XPS. The C 1s plot of Pyr-Ni-PDA also contains energies attributed to multiple chemical moieties; $284 \mathrm{eV}\left(\mathrm{CH}_{\mathrm{x}} / \mathrm{C}-\mathrm{NH}_{2}\right), 286 \mathrm{eV}(\mathrm{C}-\mathrm{O} / \mathrm{C}-\mathrm{N})$, and $288 \mathrm{eV}$ $(\mathrm{C}=\mathrm{O} / \mathrm{C}=\mathrm{N})$. The $\mathrm{O} 1 \mathrm{~s}$ region is fit with two peaks at $531 \mathrm{eV}(\mathrm{O}=\mathrm{C})$ and $533 \mathrm{eV}(\mathrm{O}-\mathrm{C})$ and 


\section{WILEY-VCH}

the $\mathrm{N} 1 \mathrm{~s}$ region is fit with three peaks assigned to $401 \mathrm{eV}$ (primary $\mathrm{R}-\mathrm{NH}_{2}$ ), $400 \mathrm{eV}$ (secondary $\mathrm{R}-\mathrm{NH}-\mathrm{R}$ ), and $399 \mathrm{eV}$ (tertiary/aromatic $=\mathrm{N}-\mathrm{R}$ ) amine functionalities. On the basis of the chemical structures of dopamine, accepted intermediate species, and the structures of polydopamine, the primary amine is associated with dopamine, the secondary amine is associated with both intermediate species and polydopamine, and the tertiary amine is attributed to tautomeric species of the intermediate species 5,6-dihydroxyindole and 5,6-indolequinone. ${ }^{[39]}$

\subsection{Biohybrid Swimming Behavior}

Once E. coli were integrated within the microtubes, their swimming behavior and trajectories (Figure 3A) were compared to free swimming bacteria (Figure 3B) over $15 \mathrm{~s}$. Free bacteria at the interface exhibit spiraling and abrupt changes in direction that are consistent with the 'run and tumble' swimming motion of $E$. coli $^{[40]}$ and is seen in the non-uniform slopes of their mean squared displacement (MSD) plots in Figure 3C. The free bacteria also had a greater average velocity, $16 \pm 4 \mu \mathrm{m} / \mathrm{s}$, than the biohybrid swimmers, allowing them to have longer trajectory paths for the given time. However, biohybrid swimmers did not display the random swimming behavior, but instead had more directional trajectories, as observed by optical microscopy tracking and confirmed by the consistency of their parabolic curves observed in their MSDs (Figure 3C). The increased directionality of the swimmers was attained by the favorable alignment and position of the bacterium inside the tube. Capture of the E. coli oriented its body length in parallel with the length of the tube, thus aligning propulsion forces and facilitating directional propulsion. Previous work from Z. Liu and K. Papadopoulos observed similar behavior of E. coli swimming in $6 \mu \mathrm{m}$ diameter capillaries, where $E$. coli exhibited limited 'run and tumble' swimming and increased unidirectional swimming. ${ }^{[41]}$ The analogous swimming behavior between E. coli in micro tubes or capillaries indicates confinement of $E$. coli restricts flagella movement, impeding the ability of the cell to reorient. The advantageous alignment of the bacteria within the tube, using PDA as an attractant, also 


\section{WILEY-VCH}

improved the swimming directionality of the biohybrid. If bacteria had adhered to the outside of the tube, their orientation would not be able to be controlled and there would be no alignment of force vectors, inhibiting swimming performance. It was noted that $E$. coli only penetrated and adhered to the narrower end of the conical tube (Figure 1A and 1B). It is hypothesized that the fabrication procedure of the tubes causes the larger opening to become partially blocked with possible residual $\mathrm{Au}$ and PDA. Although this eliminates an entry point for bacteria attachment, it is an advantage for understanding the swimming behavior of the biohybrid swimmer. Limiting the bacteria adhesion in the narrower end of the tube decreases the risk of asymmetry along the long axis and makes it simpler to study the penetration depth of bacteria into the tubes.

The degree at which the bacteria penetrated the microtube varied from one swimmer to another, therefore the length of bacteria outside the tube was labelled as the 'length of protrusion' (LOP). The LOP effects the number of bacterium flagella interacting with the tube and can alter the swimming behavior of the biohybrid swimmer. Bacteria exhibiting large LOP had significant differences in the change in angle $(\theta)$ of their tube over time $(t)$ in reference to the $x$, $y$ plane (Figure 3D) and exhibited a rocking or oscillation of the tube while swimming (Video SV3). Comparably, biohybrids with LOP $=0 \mu \mathrm{m}$ had limited changes in $\theta$ and displayed directional swimming with no rocking motion of the tube (Video SV4). Examples of the examined biohybrids with LOPs ranging from $0-4.7 \mu \mathrm{m}$ are seen in Figure 3E. The angular autocorrelation function $C(t)$ was used to quantify the changes in $\theta$ (rocking motion) in relation to biohybrid LOP by averaging the cosine of the angle change as a function of time (Equation 2 - 5). $C(t)$ values over $5 \mathrm{~s}$ for eight biohybrid swimmers with different LOPs $(0-4.7 \mu \mathrm{m})$ are shown in Figure 3F. For a ballistic, straight trajectory the microtube orientation would remain undisturbed with $C(t)=1 .{ }^{[42]}$ In the case of microtube oscillations (rocking motion), there is a decay in $C(t)$ over time, where greater oscillations elicit a more rapid decrease of $C(t)$ compared to tubes with minimal angular changes. As seen in Figure $3 \mathrm{~F}$ and inset, an increase in the 


\section{WILEY-VCH}

swimmer LOP is directly linked to a more rapid decrease in $C(t)$. In Figure $3 \mathrm{~F}$ inset a single $C(t)$ value at $\Delta t=5 \mathrm{~s}$ for each of the eight biohybrids recorded is plotted. Here, the relation of LOP to $C(t)$ over time is pronounced and indicates the $C(t)$ value may be more sensitive to LOPs between 1 and $3 \mu \mathrm{m}$. The increased rocking motion linked with larger LOP can be attributed to a greater amount of motility options for the captured bacterium. E. coli have a peritrichous arrangement of flagella and a bacterium trapped completely within the tube would have less flagella to move freely outside the tube, which would reduce the net bacterium propulsion force. For swimmers with $\mathrm{LOP}=0 \mu \mathrm{m}$, the bioactuation would be localized within the tube, promoting more net propulsion force and ballistic swimming. For swimmers with larger LOPs, the bacteria have greater freedom for flagella movement and are capable of oscillatory movements with their entire body, but are still adhered to the microtube. The variety of permitted actuation with this bacteria-interface arrangement produces greater changes in $\theta$ during swimming. The MSDs over $5 \mathrm{~s}$ of same eight swimmers were also compared to their LOP values as seen in Figure 3G. Here, there is no observed relationship between LOP and the MSD plots. The Figure 3G inset displays a single MSD value at $\Delta t=5 \mathrm{~s}$ for the swimmers, but unlike $C(t)$, the MSD values do not correlate to increased LOPs. These results indicate the LOP does not significantly contribute to the average velocity or overall translational motion of the biohybrid and that other factors are responsible for differences in the MSD values. These factors could potentially include flagella orientation and adhesion in and around the microtube as stated previously. Future research will focus on a better and more quantitative understanding of the role flagella play for microtubular biohybrid swimming. However, the relation between $C(t), \mathrm{MSD}$, and LOP gives insight for microtube biohybrid design and behavior towards their potential biomedical applications. 


\section{WILEY-VCH}

\subsection{External Motion Control of Biohybrids}

To incorporate multi-functionality into the biohybrid microswimmers, additional elements were added to the microtube fabrication. A three layer tube, Ppy-Ni-PDA, allowed the capture of a single bacterium and made it possible for the swimmer to be externally guided with a custom electromagnetic system (Figure 4 and Figure S1). This made the swimmer capable of swimming quickly to a user-defined location. A Petri dish containing the swimmers was placed in between a four-coil, electromagnetic system that could be mounted on an inverted microscope as shown in the inset in Figure 4A. The 2D trajectories of the microswimmer display a persistent motion parallel to the direction of the applied field when subjected to a constant magnetic field of approximately $8 \mathrm{mT}$; changes in the magnetic field elicit immediate changes in the swimming direction of the biohybrid swimmer (Figure 4A and Video SV5). The motion of the microtube is strongly biased according to the field direction. Previous reports have guided bacteria-driven spherical motors with external magnets, ${ }^{[26]}$ but optimization of their performance was necessary as an asymmetry of the swimmer is realized when bacteria attach to one side of a particle. Particles elicit a drag force on the bacteria and create a rotational motion around the axis decreasing the swimming directionality and translational motion. ${ }^{[28]}$ The microtube design significantly improves the directionality of the biohybrid swimmer and exhibits guided control that has not been observed with other bacteria-driven particle systems by addition of a magnetic layer.

To further improve the functions and motion control of the biohybrid microswimmer, chemical sensing capabilities of the $E$. coli were exploited by addition of a biochemical trigger to the tube. A conflict of using bacteria powered motors for medical applications is the possible risk of a bacterial infection. After the biohybrid swimmer has completed a task, a 'kill switch' could be activated to avoid bacteria attachment and reproduction in undesired locations. The hydrolysis of urea through enzymatic reaction has proven advantageous to power other micromotor systems, ${ }^{[43]}$ but also has the potential to be used as an environmental trigger to 


\section{WILEY-VCH}

inhibit bacteria mobility. In the presence of aqueous urea, urease decomposes urea: $\left(\mathrm{NH}_{2}\right)_{2} \mathrm{CO}$ $+\mathrm{H}_{2} \mathrm{O} \rightarrow \mathrm{CO}_{2}+2 \mathrm{NH}_{3}$, creating a localized distribution of $\mathrm{NH}_{3}(\mathrm{aq})$. Changes in $\mathrm{pH}$ disrupt bacteria swimming and halt micromotor movement. ${ }^{[44]}$ For a biohybrid motor with a 'kill' trigger, the two-layer biohybrid had urease immobilized on the PDA. PDA contains catechol moieties capable of covalently binding amine groups from enzymes ${ }^{[45,46]}$ allowing Pyr-PDA microtubes to be functionalized with urease by a simple incubation and rinsing procedure. $\quad E$. coli attached and swam with the urease modified tubes without hindrance (Figure 4B). The biohybrid swimmer was allowed to swim and demonstrated motility similar to the swimmers using the two-layer tubes without urease modification. Once the swimming abilities of the biohybrid were established, $50 \mathrm{mM}$ urea was added to the solution to trigger the urease in the microtubes. After $45 \mathrm{~s}$ to $1 \mathrm{~min}$, bacteria within the swimmer ceased swimming and remained immobilized for prolonged durations. The presence of $\mathrm{NH}_{3}(\mathrm{aq})$ was verified using a colorimetric assay with the indicator 4-nitrophenol (4NP), where the production of $\mathrm{NH}_{3}(\mathrm{aq})$ from the Pyr-PDA-urease tubes in the presence of urea, increased solution $\mathrm{pH}$, turning the solution yellow (Figure S2). To ensure, bacteria viability was not impacted by urea, but by $\mathrm{NH}_{3}$ (aq) production, a control study was performed (Figure 4c). Bacteria without microtubes were exposed to varying concentrations of urea and $\mathrm{NH}_{3}(\mathrm{aq})$. Addition of only urea to motility media did not hinder bacteria velocity or viability at concentrations equal or less than $50 \mathrm{mM}$ when compared to bacteria that were not exposed to any additional chemical. Addition of 1 $\mathrm{mM} \mathrm{NH}_{3}(\mathrm{aq})$ also did not impair $E$. coli viability, but higher concentrations of $\mathrm{NH}_{3}(\mathrm{aq})(10,25$, and $50 \mathrm{mM}$ ) had cell viabilities of less than $50 \%$. The urea/urease 'trigger' offers a proof-ofconcept, biocompatible solution to reducing the risk of bacterial infection with $E$. coli-driven biohybrid swimmers after completion of a task. 


\section{WILEY-VCH}

\section{Conclusion}

We have demonstrated the integration of single E. coli into microtubes to create a multifunctional biohybrid swimmer and attached a bacterium to a micro-cargo load that is not a spherical particle. The microtube was designed and tailored to accommodate a single bacterium and was modified with PDA to partially lure and trap the bacterium within. The microtubebacteria swimmer exhibited increased unidirectional swimming that has not been previously observed with other bacteria biohybrids. Swimming behavior of the bacteria-driven microtubes was characterized by analyzing bacteria penetration into the tubes and comparing changes in the tube angle and MSD. An increase in LOP of the biohybrids strongly correlated to a decline in $C(t)$ values, but showed no relation to the MSD plots indicating the LOP had little effect on overall translational motion. By incorporating 'smart' design techniques, the biohybrid swimmer became sensitive to external guidance and environmental cues. The microtubes were modified with Ni for magnetic directionality or urease to act a biocompatible 'kill switch' to the swimmer motility, making it apt for various user tasks. Future work will incorporate other swimming bacteria into the microtube chassis and study of how bacteria flagella are interacting with the tube and how the tube impacts their motility. The bacteria-driven microtubular swimmers have the potential to be the next-generation of microswimmers; incorporating improved motion directionality, net propulsion force, and multi-functionality towards minimally invasive medical applications.

\section{Experimental Section}

\section{Microtube Fabrication}

Microtubes were fabricated using a modified method previously described by Gao et al. ${ }^{[47,48]}$ A cyclopore polycarbonate membrane, containing $2 \mu \mathrm{m}$ diameter conical-shaped micropores (Catalog No 7060-2511; Whatman, Maidstone, U. K.), was used as a template to grow the microtubes. A $75 \mathrm{~nm}$ gold film was sputtered on one side of the porous membrane to create a 


\section{WILEY-VCH}

conductive surface to initiate polymer formation. An electrochemical cell was assembled where the gold coated membrane was the working electrode using aluminum foil as a contact, a Pt wire was the counter electrode, and an $\mathrm{Ag} / \mathrm{AgCl}$ with $3 \mathrm{M} \mathrm{KCl}$ was the reference electrode. Electroplating was performed with a Metrohm Autolab (Utrecht, Netherlands) PGSTAT204 Potentiostat/Galvanostat and Metrohm Autolab Nova v.1.10 software. Ppy microtubes were electropolymerized at a constant potential of $+0.8 \mathrm{~V}$ until a charge density of $0.8 \mathrm{C}$ was reached, using a plating solution of $14 \mathrm{mM} \mathrm{KNO}_{3}$ and $40 \mathrm{mM}$ pyrrole (Sigma-Aldrich) in de-ionized (DI) water. To deposit a layer of PDA within the Ppy microtubes, the membrane containing the Ppy tubes was washed with water, and incubated in $2.0 \mathrm{mg} / \mathrm{mL}$ dopamine $\mathrm{HCl}$ (Sigma-Aldrich) solution in a $10 \mathrm{mM}$ Tris buffer $(\mathrm{pH} 8.5)$ for $18 \mathrm{hrs}$ with stirring at ambient temperature. For Ppy-Ni-PDA tubes, after Ppy deposition, the pyrrole solution was removed, the membrane was washed with water, and a semiplate Ni-100 plating solution (NB Technologies GmbH, Bremen, Germany) supplemented with $0.5 \mathrm{mM}$ sodium dodecyl sulfate (SDS, Sigma-Aldrich) was added to the electrochemical cell. $\mathrm{Ni}$ was electroplated at $-1.4 \mathrm{~V}$ until a charge density of $-2.5 \mathrm{C}$ was achieved. The membrane was washed with water and incubated in a dopamine solution as described previously. After overnight PDA formation, the membrane was rinsed with water. The gold layer was removed by polishing using $5 \mu \mathrm{m}$ alumina slurry followed by rinsing with water. The membrane was dissolved in dichloromethane for 15 min to release the microtubes. Ppy-PDA tubes were collected by centrifugation at 10,000 rpm for $3 \mathrm{~min}$ and washed and resuspended with fresh dichloromethane. Centrifugation and washing was repeated two more times with dichloromethane, two times with isopropanol, and finally two times with ultrapure water (18.2 $\mathrm{M} \Omega \mathrm{cm})$. The magnetic Ppy-Ni-PDA tubes were separated from washing solutions using a magnet instead of centrifugation. Ppy-PDA and Pyr-Ni-PDA microtubes were stored in nanopure water at room temperature. For Ppy-PDA-urease tubes, Ppy-PDA tubes that had been separated from the membrane scaffold as described previously, were incubated in a $0.3 \mathrm{mg} / \mathrm{mL}$ urease (Canavalia ensiformis (Jack bean), Simga-Aldrich) in PBS for $1.5 \mathrm{hrs}$ with shaking. 


\section{WILEY-VCH}

Ppy-PDA-Urease tubes were centrifuged at 12,000 rpm for $5 \mathrm{~min}$ and washed twice with fresh PBS, resuspended in PBS, and stored at $4{ }^{\circ} \mathrm{C}$.

\section{Bacteria Culture}

Escherichia coli (E. coli) MG1655 cultured on LB agar plates (Sigma-Aldrich) were transferred to $5 \mathrm{~mL} \mathrm{LB}$ broth (Sigma-Aldrich) and allowed to divide overnight at $37^{\circ} \mathrm{C}$ and $150 \mathrm{rpm}$. $0.5 \mathrm{~mL}$ of concentrated MG1655 solution was diluted in $5 \mathrm{~mL}$ of fresh LB broth and allowed to culture another 1.5-2 hrs until the measured optical density at $600 \mathrm{~nm}\left(\mathrm{OD}_{600}\right)$ using a BioTek (Bad Friedrichshall, Germany) Gen5 Synergy 2 plate reader was $\sim 0.2$. The $\mathrm{OD}_{600}$ was obtained from $200 \mu \mathrm{L}$ of media with bacteria in a 96-well plate at ambient temperature using bacteria free media for a control. Bacteria were centrifuged (6000 rpm, $3 \mathrm{~min})$ and resuspended twice in motility media $\left(0.01 \mathrm{M} \mathrm{K}_{3} \mathrm{PO}_{4}, 67 \mathrm{mM} \mathrm{NaCl}, 0.1 \mathrm{mM}\right.$ ethylenediaminetetraacetic acid (EDTA), 0.002\% v/v Tween-20, 0.01 M D-(+)-glucose, $\mathrm{pH}$ adjusted to 7.4). All experiments with bacteria were performed in motility media unless specified.

\section{Biohybrid Microswimmer Formation}

Microtubes were added to bacteria in motility media and allowed to incubate at $37^{\circ} \mathrm{C}$ and 150 rpm for $30 \mathrm{~min}$. The solutions containing the biohybrid swimmers were imaged in petri dishes with glass coverslip bottoms (Cellview Cell Culture Dish, Greiner Bio-One) at ambient temperature. Videos and images were acquired using bright field microscopy with an inverted Leica (Wetzlar, Germany) DMI3000B microscope, Leica DFC3000G camera, and Leica Application Suite v.4.5.0 software. For magnetically guided microswimmers, motion control experiments were conducted using an electromagnetic coil system. The system consisted of four orthogonally oriented iron-core electromagnets. The coils were built onto a custom-made microscope stage to surround a $35 \mathrm{~mm}$ Petri dish and was placed onto an inverted Zeiss (Carl Zeiss, Inc., Oberkochen, Germany) Axio Observer A1 microscope with a 20x objective (Figure 4 and Figure S1). The input current for the coils was controlled by motor drivers and an Arduino microcontroller board, and the $\sim 8 \mathrm{mT}$ magnetic field strength was calibrated using 


\section{WILEY-VCH}

a Lake Shore Cryotronics (Darmstatdt, Germany) Model 460-3-Channel Gaussmeter.

Microswimmers were recorded with a Zeiss Axiocam 503 CCD camera.

Live/Dead Assay with Urea and $\mathrm{NH}_{3}(a q)$

Bacteria suspended in motility media at $\mathrm{OD}_{600}=0.2$ were incubated various concentrations of urea or $\mathrm{NH}_{3}$ (aq) $(1,10,25$, or $50 \mathrm{mM}$ ) for $15 \mathrm{~min}$ at room temperature. The control sample had no exposure to either chemical. Bacteria were centrifuged at $6000 \mathrm{rpm}$ for $3 \mathrm{~min}$ and resuspended in fresh motility media. Cells were incubated with $2 \mu \mathrm{L} / \mathrm{mL}$ of propidium iodide and STYO 9 (Life Technologies) for $15 \mathrm{~min}$. Cells were centrifuged, washed twice with motility media, and immediately imaged with fluorescent microscopy. Percent cell viability was defined as the total number of live cells divided by the sum of live and dead cells.

Scanning Electron Microscopy (SEM)

Bacteria and tubes were imaged with a Zeiss Ultra 55 Gemini scanning electron microscope using an accelerating voltage of $5 \mathrm{keV}$ and an in-lens detector. To prepare samples for SEM, biohybrids suspended in motility media were allowed to sediment on clean, plasma etched (1 min argon plasma, Diener Electronic Atto Plasma Cleaner, Ebhausen, Germany) silicon wafer chips (5 x $6 \mathrm{~mm}$ ) for $1 \mathrm{hr}$ at ambient temperature. Wafers were incubated in $2.5 \%$ glutaraldehyde in PBS for $45 \mathrm{~min}$ at $4{ }^{\circ} \mathrm{C}$, rinsed with PBS, then water. Bacteria were dehydrated in a series of increasing aqueous ethanol concentrations $(30 \%, 50 \%, 70 \%, 90 \%$, and $100 \%$ ) for $5 \mathrm{~min}$ in each solution and $10 \mathrm{~min}$ in pure ethanol. Bacteria were further dehydrated and preserved using a series of hexamethyldisilazane (HMDS, Sigma-Aldrich) solutions; 2:1 Ethanol:HMDS (15 min), 1:2 Ethanol: HMDS (15 min), pure HMDS (15 min). Wafers and bacteria air dried followed by sputtering deposition of $3 \mathrm{~nm}$ gold using a Bal-tec MED 020 coating system (Leica). Microtubes without bacteria needed no additional preparation before imaging. 


\section{WILEY-VCH}

$X$-ray Photoelectron Spectroscopy (XPS)

XPS analysis was performed with a Thermo VG Thetaprobe 300 (Thermo Fischer Scientific) system using monochromatic incident $\mathrm{Al} \mathrm{K \alpha}$ radiation $(h v=1486.68 \mathrm{eV}$; spot size $400 \mu \mathrm{m}$; base pressure $<10^{-7} \mathrm{~Pa}$; average detection angle of $53^{\circ}$ with respect to the sample surface). Energy calibration, removal of contaminants, and charge compensation during the measurements were carried out. The XPS spectra were deconvoluted with the Thermo VG Scientific Advantage 5.47 software (Thermo Fischer Scientific). Samples were prepared by air drying microtubes in DI water onto silicon wafer chips (5 x $6 \mathrm{~mm})$.

\section{Bacteria and Biohybrid Swimmer Tracking and Analysis}

Tracking of bacterium-driven microtube swimmers and bacteria was performed on the recorded videos to compute the velocity and mean square displacement (MSD) using an in-house developed script in Python with the OpenCV library. The length of the videos recorded was between 15 and $60 \mathrm{~s}$. Thresholding criteria was used to distinguish bacteria and tubes from the background with their positions taken as the center of mass of the contour. Bayesian decision making determined the trajectory of the motors over time. The average velocities calculated are an average of a minimum of 10 biohybrids or bacteria with the error represented as the standard deviation. The MSD was calculated using Equation 1,

$$
\operatorname{MSD}(t)=\left\langle r^{2}(t)\right\rangle=\left\langle\left(\vec{r}\left(t_{0}+t\right)-\vec{r}\left(t_{0}\right)\right)^{2}\right\rangle_{t_{0}}
$$

where $\vec{r}$ is a two-dimensional position vector and averaging over a given time $(t)$.

To calculate the angular autocorrelation, $\mathrm{C}(\mathrm{t})$, for the swimmer, the orientation and angle was computed from the elongated shape $(S)$ of the microtube using image moments in reference to the $x, y$ plane of the image. We defined the major and minor axis of a non-symmetric shape as the axis along which the pixel variance is maximum and minimum, respectively. The tube orientation was taken as the major axis, with an angle $(\theta)$ given by:

$$
\tan \theta=\frac{2 \sigma_{11}}{\sigma_{20}-\sigma_{02}}
$$




\section{WILEY-VCH}

where $\sigma_{11}$ corresponds to the covariance, $\sigma_{20}$ to the variance along the $x$ axis, and $\sigma_{02}$ to the variance along the $y$ axis. The central image moments $\left(\sigma_{n m}\right)$ were computed on a binary image which included the shape of the tube, with their general expression being:

$$
\begin{gathered}
\sigma_{n m}=\frac{\sum \sum_{x, y \in S}\left(x-m_{10}\right)^{n}\left(y-m_{01}\right)^{m}}{\text { Area of } S} \\
m_{i j}=\frac{\sum \sum_{x, y \in S} x^{i} y^{i}}{\text { Area of } S}
\end{gathered}
$$

With the obtained angle information, it was possible to characterize the change in orientation of the tubes by using the angular autocorrelation function, $C(t)$. The orientation was defined by the unitary vector $\hat{v}(t)$ with angle $\theta(t)$ and $C(t)$ was computed with the following formula:

$$
C(t)=\left\langle\hat{v}\left(t_{0}+t\right) \cdot \hat{v}\left(t_{0}\right)\right\rangle_{t 0}=\left\langle\cos \left(\theta\left(t+t_{0}\right)-\theta(t)\right)\right\rangle_{t 0}
$$

\section{Supporting Information}

Supporting Information is available from the Wiley Online Library or from the author.

\section{Acknowledgements}

The research leading to these results was achieved thanks to the financial support from the European Research Council for the European Union's Seventh Framework Program (FP7/2007-2013) starting Grant no. 311529 (Lab-in-a-tube and Biosensors) and the Grassroots Initiative funding from the Max Planck Institute for Intelligent Systems. The authors also thank Mr. Alejandro Posada for designing the graphics in Figure 1 and Mr. Jiang Zhuang for assisting with the magnetic guidance experiments.

Received: ((will be filled in by the editorial staff))

Revised: ((will be filled in by the editorial staff)) Published online: ((will be filled in by the editorial staff)) 


\section{WILEY-VCH}

\section{References}

[1] J. Xi; J. J. Schmidt; C. D. Montemagno, Nat Mater. 2005, 4, 180.

[2] B. Behkam; M. Sitti, Appl. Phys. Lett. 2007, 90, 023902.

[3] R. W. Carlsen; M. Sitti, Small. 2014, 10, 3831.

[4] S. Sánchez; L. Soler; J. Katuri, Angew. Chem. Int. Ed. 2015, 54, 1414.

[5] J. Parmar; X. Ma; J. Katuri; J. Simmchen; M. M. Stanton; C. Trichet-Paredes; L. Soler; S. Sanchez, Sci. Tech. Adv. Mater. 2015, 16.

[6] H. Wang; M. Pumera, Chem. Rev. 2015, 115, 8704.

[7] M. M. Stanton; C. Trichet-Paredes; S. Sanchez, Lab Chip. 2015, 15, 1634.

[8] R. Raman; C. Cvetkovic; S. G. M. Uzel; R. J. Platt; P. Sengupta; R. D. Kamm; R. Bashir, Proc. Natl. Acad. Sci. U.S.A. 2016, 113, 3497.

[9] Y. Tanaka; K. Sato; T. Shimizu; M. Yamato; T. Okano; T. Kitamori, Lab Chip. 2007, 7, 207.

[10] B. J. Williams; S. V. Anand; J. Rajagopalan; M. T. A. Saif, Nat Commun. 2014, 5.

[11] S.-J. Park; M. Gazzola; K. S. Park; S. Park; V. Di Santo; E. L. Blevins; J. U. Lind; P. H. Campbell; S. Dauth; A. K. Capulli; F. S. Pasqualini; S. Ahn; A. Cho; H. Yuan; B. M. Maoz; R. Vijaykumar; J.-W. Choi; K. Deisseroth; G. V. Lauder; L. Mahadevan; K. K. Parker, Science. 2016, 353, 158.

[12] T. H. M. P. Consortium, Nature. 2012, 486, 207.

[13] J. Zhuang; M. Sitti, Sci. Rep. 2016, 6, 32135.

[14] R. Di Leonardo; L. Angelani; D. Dell'Arciprete; G. Ruocco; V. Iebba; S. Schippa; M. P. Conte; F. Mecarini; F. De Angelis; E. Di Fabrizio, Proc. Natl. Acad. Sci. U.S.A. 2010, 107, 9541.

[15] Y. Hiratsuka; M. Miyata; T. Tada; T. Q. P. Uyeda, Proc. Natl. Acad. Sci. U.S.A. 2006, $103,13618$.

[16] M. Kojima; Z. Zhang; M. Nakajima; K. Ooe; T. Fukuda, Sens. Actuators B Chem. 2013, $183,395$.

[17] S. Taherkhani; M. Mohammadi; J. Daoud; S. Martel; M. Tabrizian, ACS Nano. 2014, 8, 5049 .

[18] O. Felfoul; M. Mohammadi; S. Taherkhani; D. de Lanauze; Y. Zhong Xu; D. Loghin; S. Essa; S. Jancik; D. Houle; M. Lafleur; L. Gaboury; M. Tabrizian; N. Kaou; M. Atkin; T. Vuong; G. Batist; N. Beauchemin; D. Radzioch; S. Martel, Nat Nano. 2016, advance online publication.

[19] A. V. Singh; M. Sitti, Adv. Healthc. Mater. 2016, n/a.

[20] A. Sahari; M. A. Traore; B. E. Scharf; B. Behkam, Biomed. Microdevices. 2014, 16,

[21] D. Kim; A. Liu; E. Diller; M. Sitti, Biomed. Microdevices. 2012, 14, 1009.

[22] R. Fernandes; M. Zuniga; F. R. Sassine; M. Karakoy; D. H. Gracias, Small. 2011, 7,

[23] S. J. Park; H. Bae; J. Kim; B. Lim; J. Park; S. Park, Lab Chip. 2010, 10, 1706.

[24] B. Behkam; M. Sitti, Appl. Phys. Lett. 2008, 93, 223901.

[25] S. Martel; C. C. Tremblay; S. Ngakeng; G. Langlois, Appl. Phys. Lett. 2006, 89.

[26] M. M. Stanton; J. Simmchen; X. Ma; A. Miguel-López; S. Sánchez, Adv. Mater. Interf. 2016, 3, n/a.

[27] V. Arabagi; B. Behkam; E. Cheung; M. Sitti, J. Appl. Phys. 2011, 109, 114702.

[28] A. Sahari; D. Headen; B. Behkam, Biomed. Microdevices. 2012, 14, 999.

[29] V. Magdanz; M. Guix; F. Hebenstreit; O. G. Schmidt, Adv. Mater. 2016, 28, 4084.

[30] I. S. M. Khalil; V. Magdanz; S. Sanchez; O. G. Schmidt; S. Misra, Journal of MicroBio Robotics. 2014, 9, 79.

[31] V. Magdanz; S. Sanchez; O. G. Schmidt, Adv. Mater. 2013, 25, 6581. 


\section{WILEY-VCH}

[32] V. Magdanz; M. Medina-Sánchez; Y. Chen; M. Guix; O. G. Schmidt, Adv. Funct. Mater. 2015, 25, 2763.

[33] F. R. Blattner; G. Plunkett; C. A. Bloch; N. T. Perna; V. Burland; M. Riley; J. ColladoVides; J. D. Glasner; C. K. Rode; G. F. Mayhew; J. Gregor; N. W. Davis; H. A. Kirkpatrick; M. A. Goeden; D. J. Rose; B. Mau; Y. Shao, Science. 1997, 277, 1453.

[34] R. Di Leonardo; D. Dell'Arciprete; L. Angelani; V. Iebba, Phys. Rev. Lett. 2011, 106, 038101.

[35] H. Lee; S. M. Dellatore; W. M. Miller; P. B. Messersmith, Science. 2007, 318, 426.

[36] N. B. Arnfinnsdottir; V. Ottesen; R. Lale; M. Sletmoen, PLoS ONE. 2015, 10.

[37] K. Sun; Y. Xie; D. Ye; Y. Zhao; Y. Cui; F. Long; W. Zhang; X. Jiang, Langmuir. 2012, $28,2131$.

[38] S. Rozhok; C. K. F. Shen; P.-L. H. Littler; Z. Fan; C. Liu; C. A. Mirkin; R. C. Holz, Small. 2005, 1, 445.

[39] R. A. Zangmeister; T. A. Morris; M. J. Tarlov, Langmuir. 2013, 29, 8619.

[40] E. Lauga; W. R. DiLuzio; G. M. Whitesides; H. A. Stone, Biophys. J. 2006, 90, 400.

[41] Z. Liu; K. D. Papadopoulos, Appl. Environ. Microbiol. 1995, 61, 3567.

[42] Carsten Krüger; G. Klös; C. Bahr; C. C. Maass, arXiv. 2016, eprint arXiv:1605.03396.

[43] X. Ma; X. Wang; K. Hahn; S. Sánchez, ACS Nano. 2016, 10, 3597.

[44] J. Zhuang; R. W. Carlsen; M. Sitti, Sci. Rep. 2015, 5.

[45] L. Zhou; Y. Jiang; L. Ma; Y. He; J. Gao, Appl. Biochem. Biotechnol. 2015, 175, 1007.

[46] H. Lee; J. Rho; P. B. Messersmith, Adv. Mater. 2009, 21, 431.

[47] W. Gao; S. Sattayasamitsathit; J. Orozco; J. Wang, JACS. 2011, 133, 11862.

[48] W. Gao; S. Sattayasamitsathit; A. Uygun; A. Pei; A. Ponedal; J. Wang, Nanoscale. 2012, 4, 2447. 


\section{WILEY-VCH}

\section{Figures}

A
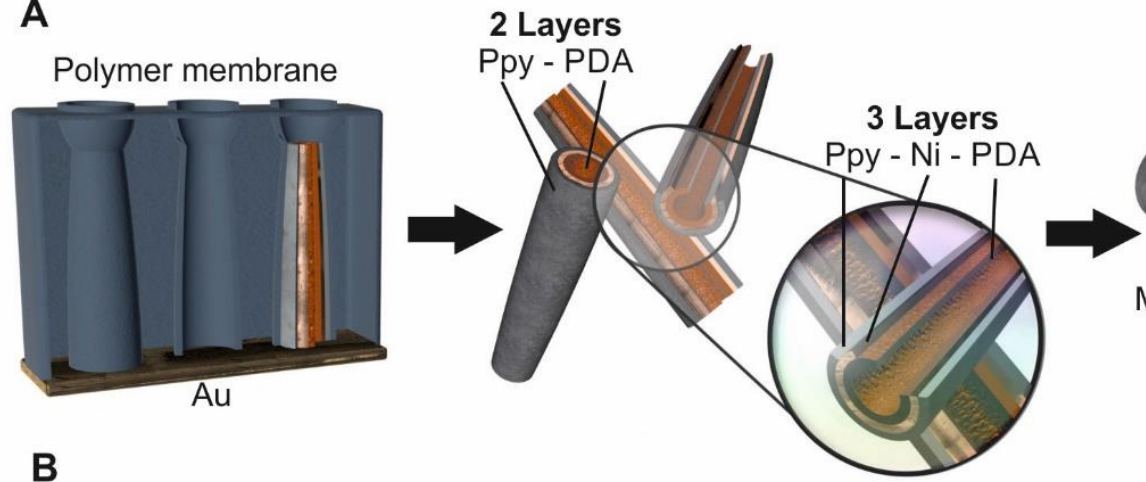

B
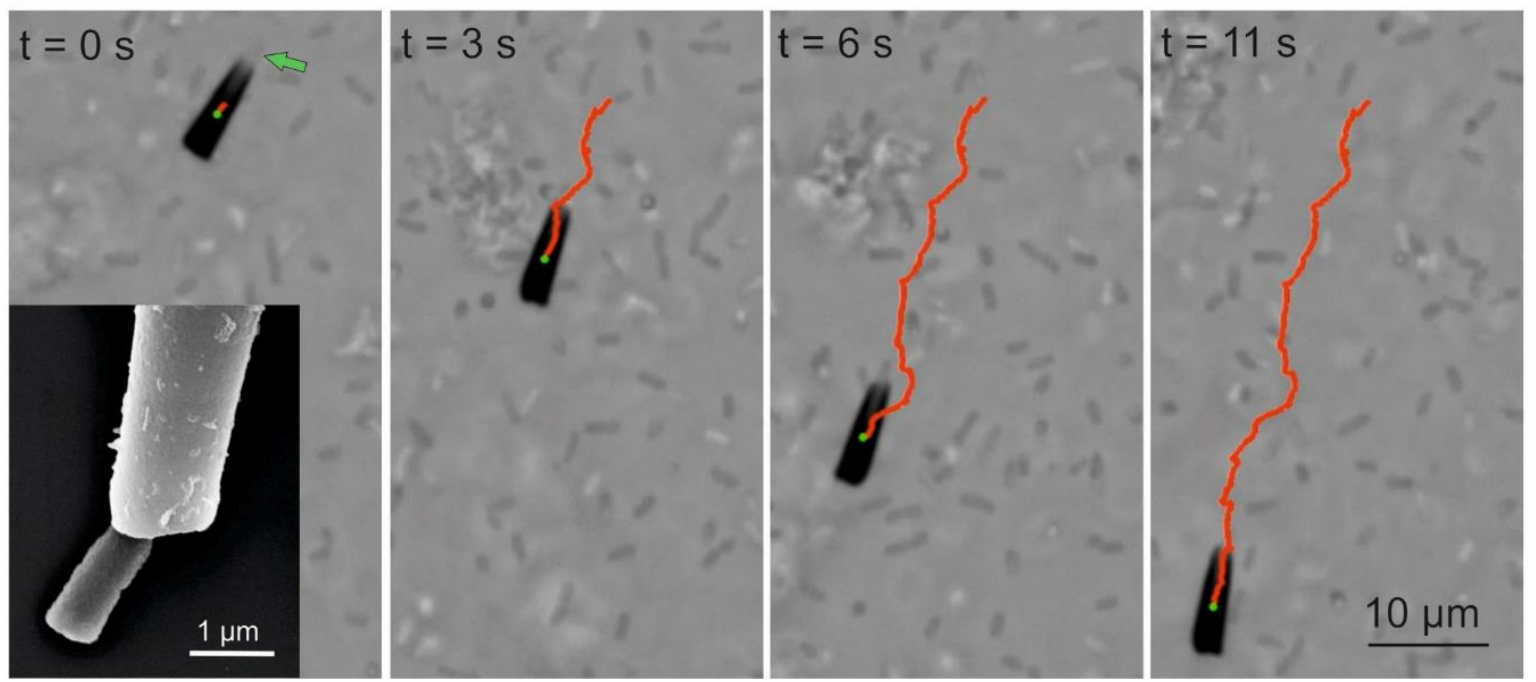

Figure 1. Bacteria-driven microtubular microswimmer concept. (A) Ppy microtubes were grown electrochemically in a membrane scaffold modified with a Au base layer. Tubes had PDA deposited on the inner wall (two layers) or Ni was electrochemically plated followed by deposition of PDA (three layers). Tubes were released from scaffold and incubated with E. coli to create a biohybrid microswimmer. (B) Example of bacterium-driven swimming over time. Green arrow in first panel indicates position of single E. coli partially contained within the tube. Inset displays SEM image of the microtube swimmer with the attached bacterium. 


\section{WILEY-VCH}
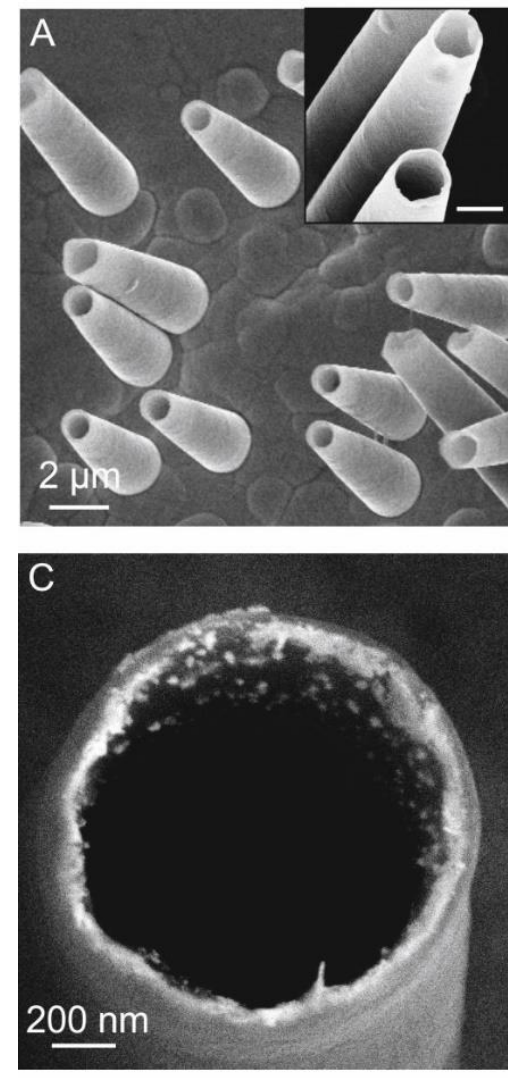
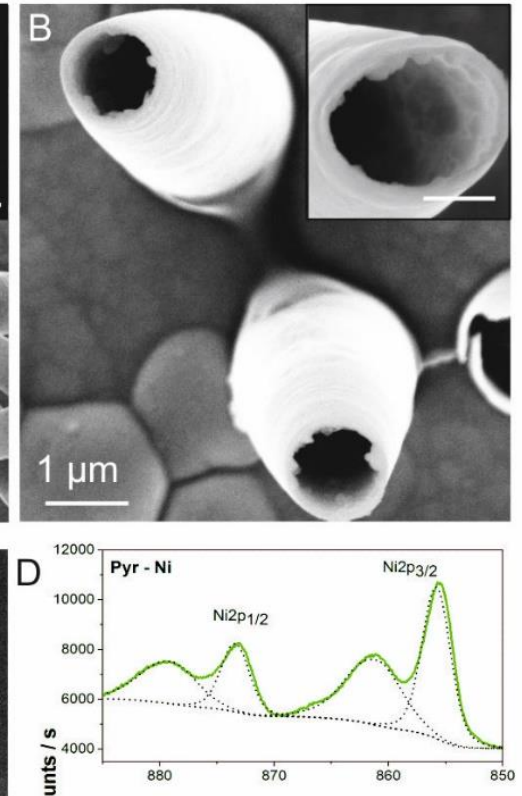

ठั.

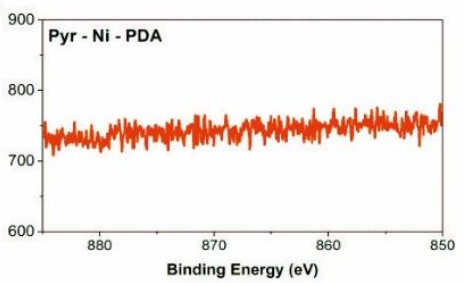

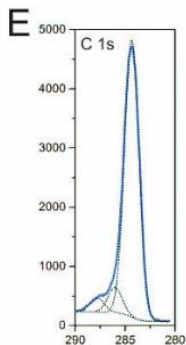
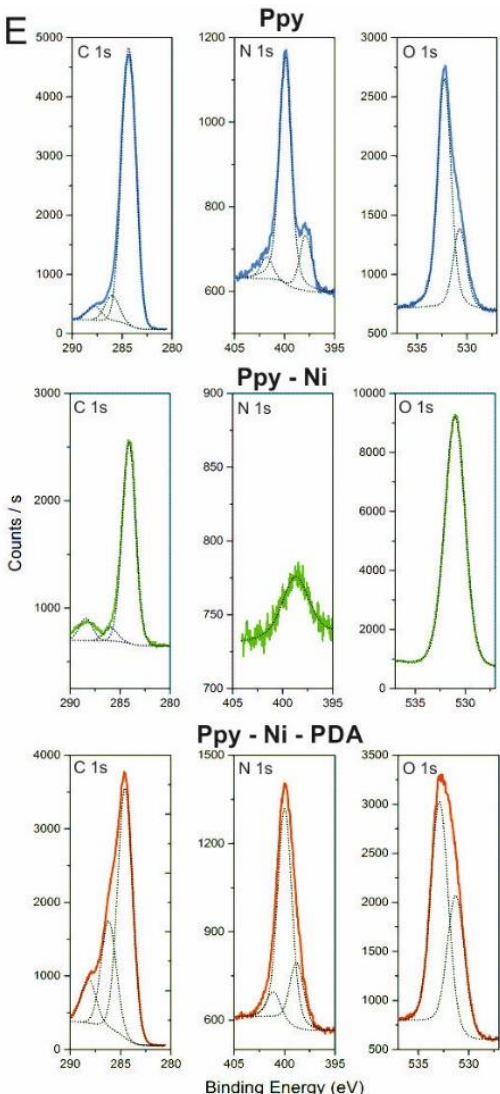

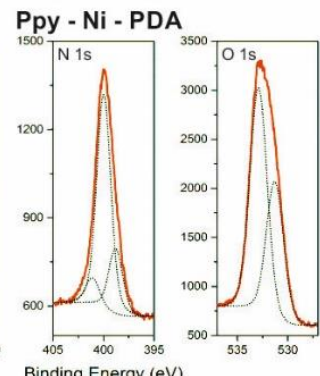

Figure 2. SEM images of polymer microtubes and XPS data. (A) Ppy microtubes grown on a $\mathrm{Au}$-coated membrane. Inset displays increased magnification of tubes. Inset scale bar $=1 \mu \mathrm{m}$. (B) Ppy microtubes with PDA adhered within the tubes. Inset displays increased magnification inside the tube and PDA nanostructure formation. Inset scale bar $=500 \mathrm{~nm}$. (C) Ppy tube with Ni nanoparticles within the interior of the tube for magnetic guidance. (D) XPS of Pyr-Ni tubes, as shown in image $c$, and XPS of Pyr-Ni-PDA tubes. (e) XPS data for Pyr, Pyr-Ni, and Pyr-NiPDA tubes for C 1s, N 1s, and O 1s peaks. Deconvolution of XPS data is shown in black dashed lines in spectra. 


\section{WILEY-VCH}
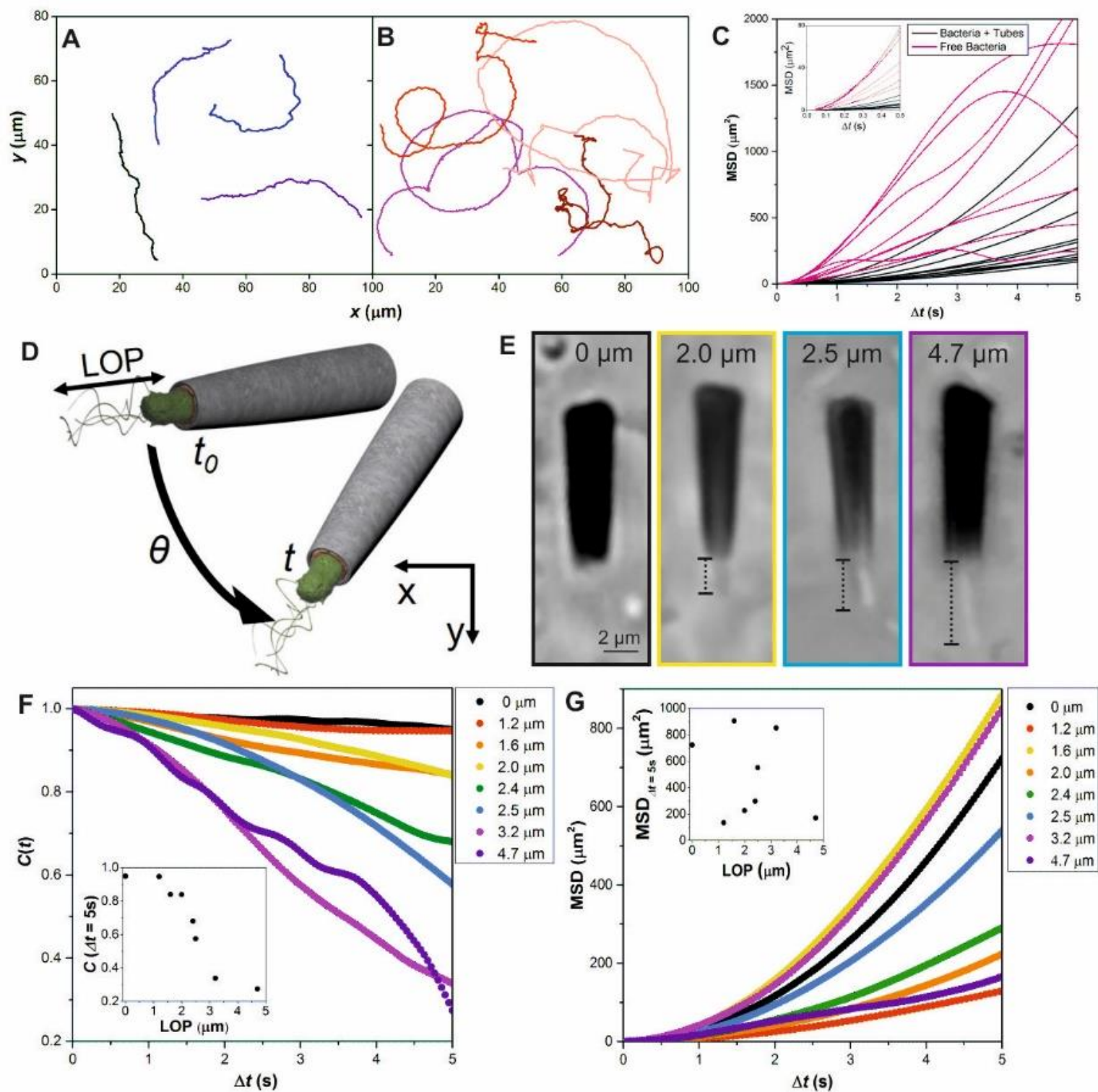

Figure 3. Quantitative analysis of biohybrid microswimmer motion behavior. (A) Swimming tracks of four biohybrid swimmers compared to (B) four free bacteria obtained over $15 \mathrm{~s}$. All tracks were obtained from different bacteria and biohybrid swimmers. (C) MSD plots of biohybrid (black) and free bacteria (pink) over 5 seconds. Inset displays parent graph with increased magnification from $0-0.5 \mathrm{~s}$. (D) Schematic of change in angle $(\theta)$ during biohybrid swimming over time $(t)$ and length of protrusion (LOP) of bacteria from microtube. (E) Examples of biohybrid LOPs (0, 2.0, 2.5, and $2.7 \mu \mathrm{m})$ analyzed in Figs. 3f and 3g. Dotted lines portray approximate length of bacteria protruding from tube. (F) $C(t)$ plots calculated over $5 \mathrm{~s}$ from biohybrid swimmers with varied LOPs $(0-4.7 \mu \mathrm{m})$. Inset displays $C(t)$ values at $\Delta t=5 \mathrm{~s}$ for each type of biohybrid LOP in parent graph. (G) MSD plots calculated over $5 \mathrm{~s}$ from biohybrids with varied LOPs $(0-4.7 \mu \mathrm{m})$. Inset displays MDS values at $\Delta t=5 \mathrm{~s}$ for each type of biohybrid LOP in parent graph. 


\section{WILEY-VCH}
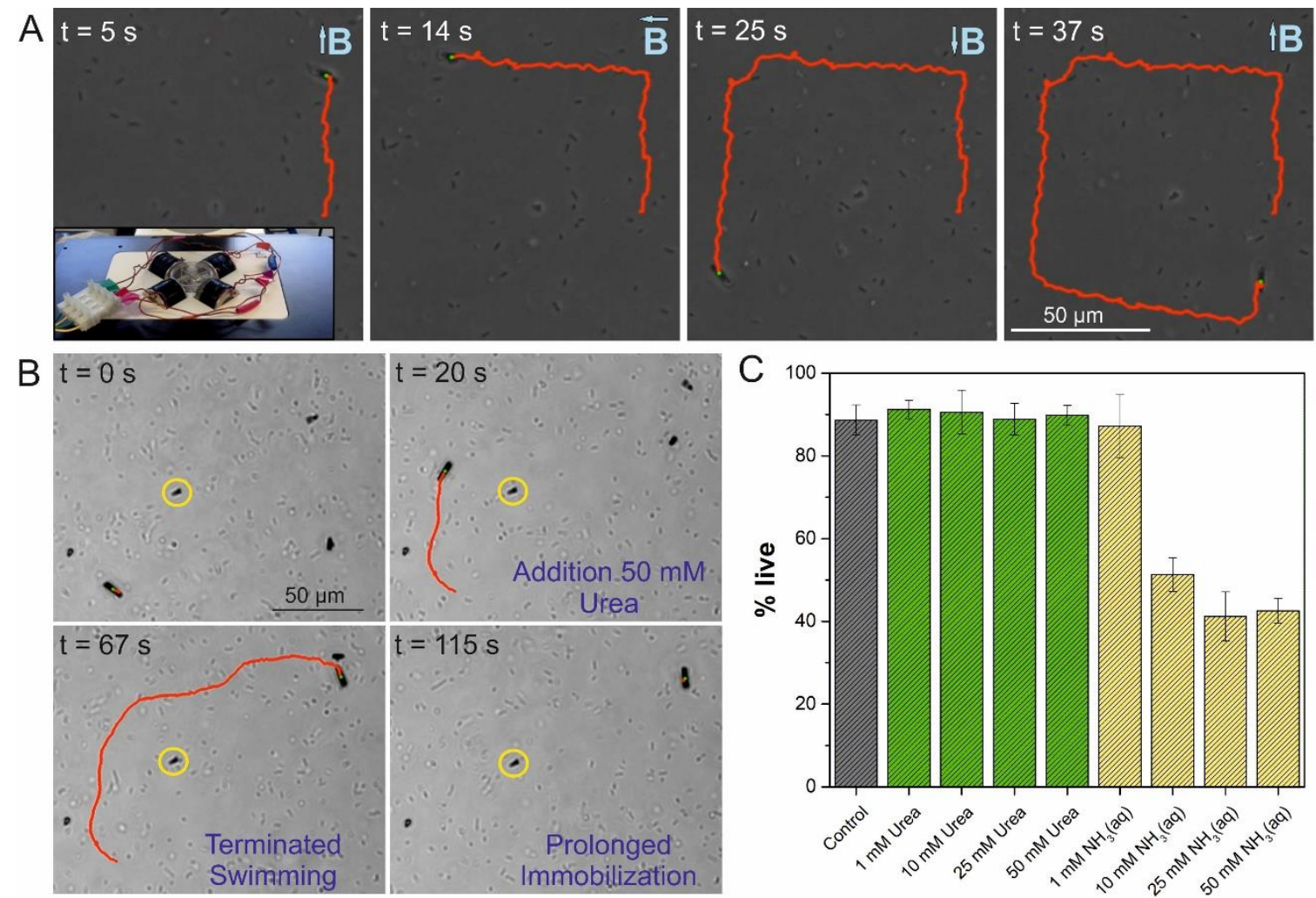

Figure 4. External motion control of biohybrid swimmers. (A) Magnetic guidance of a PyrNi-PDA bacterium-driven microtube swimmer. Direction of magnetic field $(B)$ is shown with the arrow. Inset displays a custom magnetic coil setup on an optical microscope stage. (B) PyrPDA-Urease tubes trapped single bacteria and were able to swim freely. Upon addition of $50 \mathrm{mM}$ urea, the motility of the biohybrid swimmer was terminated due to localized formation of $\mathrm{NH}_{3}$ (aq) and no further swimming was observed. The yellow circle indicates immobile particle as a visual reference. (C) Viability assay of bacteria incubated in various concentrations $\left(1,10,25\right.$, or $50 \mathrm{mM}$ ) of urea (green) or $\mathrm{NH}_{3}$ (aq) (yellow). Control is displayed in grey. 


\section{WILEY-VCH}

\section{ToC}

For a new biohybrid microswimmer design, single swimming E. coli bacteria are captured inside polydopamine modified microtubes. Bacteria flagella drive the microtube through liquid media and demonstrate directional swimming that can be terminated with a chemically activated switch. Swimming behavior is investigated to understand its feasibility of potential future biomedical applications.

Keywords: biohybrid, polydopamine, micromotor, microswimmer, E. coli

Morgan M. Stanton, Byung-Wook Park, Albert Miguel-López, Xing Ma, Metin Sitti*, Samuel Sánchez*

Biohybrid Microtube Swimmers Driven by Single Captured Bacteria

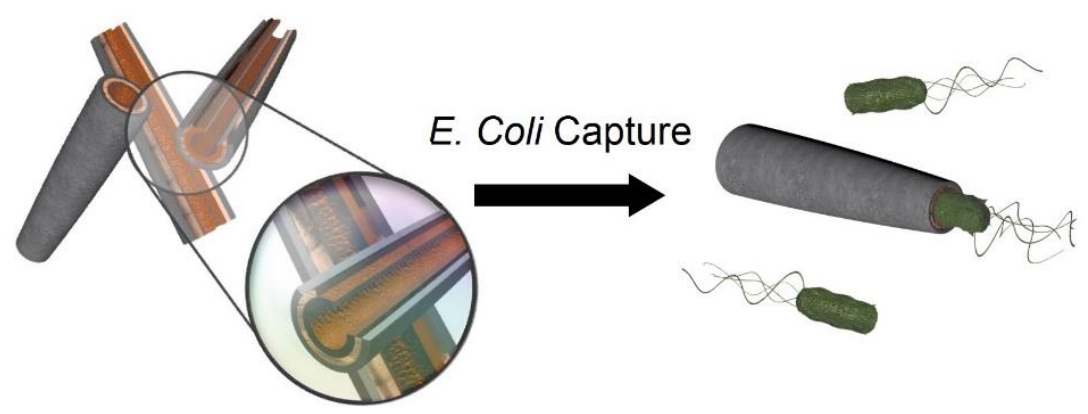




\section{WILEY-VCH}

Copyright WILEY-VCH Verlag GmbH \& Co. KGaA, 69469 Weinheim, Germany, 2016.

\section{Supporting Information}

\section{Biohybrid Microtube Swimmers Driven by Single Captured Bacteria}

Morgan M. Stanton, Byung-Wook Park, Albert Miguel-López, Xing Ma, Metin Sitti*, Samuel Sánchez*

Video SV1: E. coli swimming with two layer, Pyr-PDA microtube (60x magnification).

Video SV2: E. coli swimming with two layer, Pyr-PDA microtube (20x magnification).

Video SV3: Biohybrid (LOP = 2.7) exhibiting a rocking motion while swimming $(60 \mathrm{x}$ magnification).

Video SV4: Biohybrid (LOP $=0 \mu \mathrm{m})$ exhibiting ballistic swimming with no rocking motion (40x magnification).

Video SV5: E. coli swimming with three layer, Pyr-Ni-PDA microtube being externally guided with magnetic coils (20x magnification).

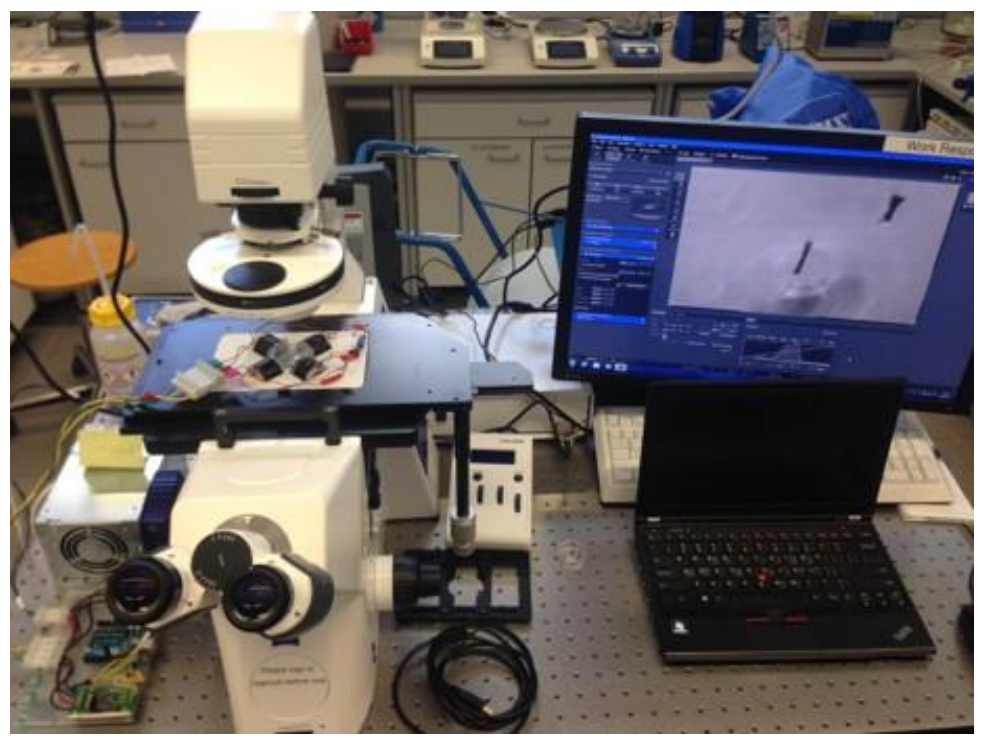

Figure S1. Experimental setup for magnetic guidance. A custom electromagnetic coil system with iron-core electromagnets with a magnetic field strength of $\sim 8 \mathrm{mT}$ surround a Petri dish containing bacteria microswimmers. 


\section{WILEY-VCH}

Determination of $\mathrm{NH}_{3}(\mathrm{aq})$ Production

The colorimetric indicator, 4-nitrophenol (4NP, Sigma-Aldrich), was used for qualitative determination of the presence of $\mathrm{NH}_{3}(\mathrm{aq})$. Aqueous 4NP remains colorless below $\mathrm{pH} 7.5$, but at higher $\mathrm{pH}$ values, turns the solution a pale yellow. Addition of urea to a solution of urease modified microtubes (Pyr-PDA-urease) produced $\mathrm{NH}_{3}(\mathrm{aq})$, increased solution $\mathrm{pH}$, and inhibited bacteria motility. To validate the $\mathrm{NH}_{3}(\mathrm{aq})$ formation was due to the catalysis of the urea with the Pyr-PDA-urease tubes, negative and positive controls were examined with 4NP as seen in Figure $\mathbf{S 2}$.

Five, $1 \mathrm{~mL}$ solutions were compared in transparent cuvettes. All samples were prepared with DI water and contained $0.4 \% \mathrm{w} / \mathrm{v} 4 \mathrm{NP}$ as a $\mathrm{pH}$ indicator. Samples $1-3$ were negative controls and indicated no change in $\mathrm{pH}$ and therefore no $\mathrm{NH}_{3}(\mathrm{aq})$ production. Sample 1 had no additional chemicals or components to the 4NP, Sample 2 contained $50 \mathrm{mM}$ of urea, and Sample 3 contained Pyr-PDA-urease microtubes $(\sim 60$ tubes/10 $\mu \mathrm{L})$. As a positive control, $20 \mathrm{mM}$ of $\mathrm{NH}_{3}(\mathrm{aq})$ was added to the Sample 4 cuvette, increasing the $\mathrm{pH}$ and turning the solution yellow. In Sample 5, $50 \mathrm{mM}$ of urea was added to the Pyr-PDA-urease microtubes ( $\sim 60$ tubes $/ 10 \mu \mathrm{L}$ ) soltuion, triggering the catalysis and formation of $\mathrm{NH}_{3}(\mathrm{aq})$. The production of the $\mathrm{NH}_{3}(\mathrm{aq})$ changed the solution to from colorless to yellow 15 seconds after the addition of urea. The change in color of the solution confirms the presence of $\mathrm{NH}_{3}(\mathrm{aq})$ and that its formation is due to the urease microtube and urea catalysis reaction. 


\section{WILEY-VCH}

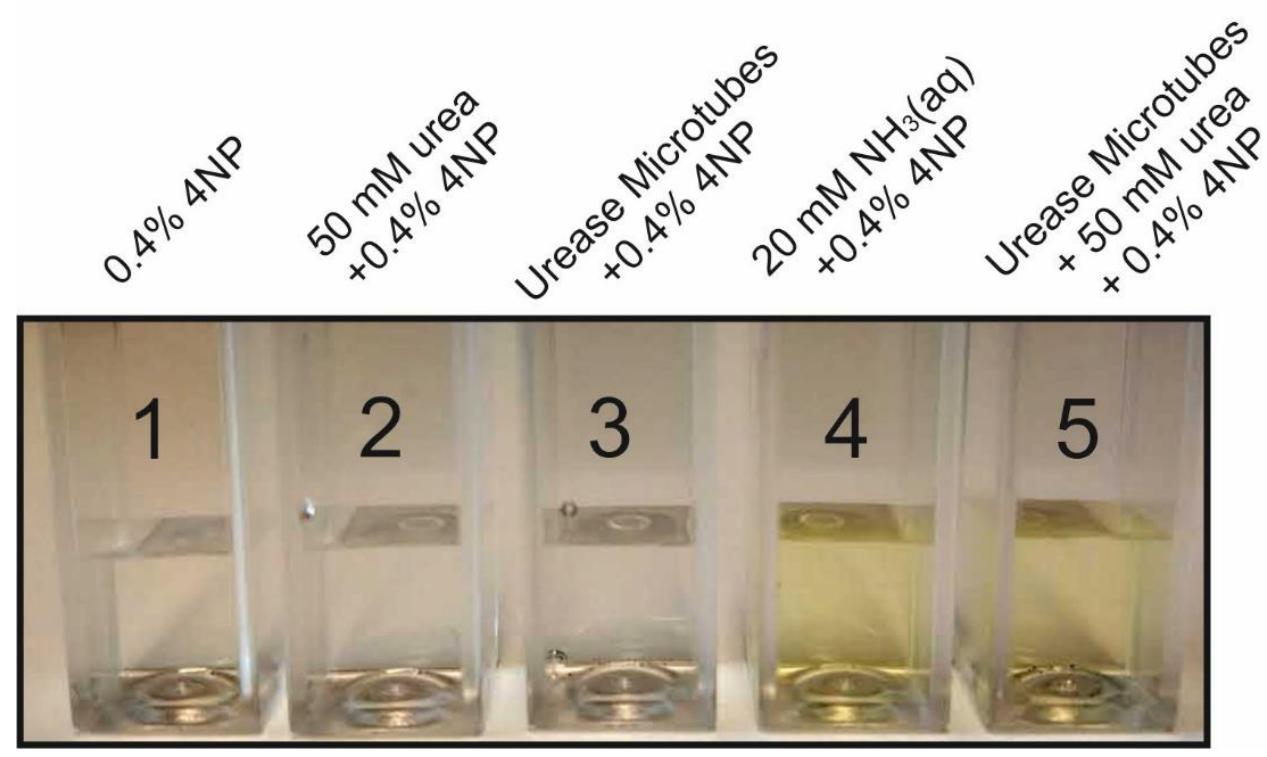

Figure S2. Qualitative determination of $\mathrm{NH}_{3}(\mathbf{a q})$ formation from urease modified microtubes (Pyr-PDA-urease) using 4NP as a $\mathrm{pH}$ indicator. 